\title{
ION-IMPLANTED GLASS WAVEGUIDES: A REVIEW
}

\author{
${ }^{1,2}$ Chun-Xiao Liu, ${ }^{2,3}$ Bo Peng, ${ }^{2,3}$ Wei Wei, \\ ${ }^{2}$ Wei-Nan Li, ${ }^{2}$ Hai-Tao Guo and ${ }^{4}$ Shu Cheng \\ ${ }^{1}$ School of Electronic and Information Engineering, \\ Hefei Normal University, Hefei 230601, China \\ ${ }^{2}$ State Key Laboratory of Transient Optics and Photonics, \\ $\mathrm{Xi}^{\prime}$ an Institute of Optics and Precision Mechanics, \\ Chinese Academy of Sciences (CAS), Xi'an 710119, China \\ ${ }^{3}$ Key Laboratory for Organic Electronics and Information Displays (KLOEID), \\ Instit ute of Advanced Materials (IAM), \\ Nanjing University of Posts and Telecommunications, Nanjing 210046, China \\ ${ }^{4}$ Changchun University of Science and Technology, Changchun 130022, China
}

Received 2013-01-08, Revised 2013-04-06; Accepted 2013-05-20

\begin{abstract}
As a well-known technique for material modification, ion implantation has become an effective method to fabricate waveguide in optical glasses. The guiding structures can be accurately controlled by the adjustment of the species, energies and fluences of the implanted ions. In the present work, we review the results of up-to-date research on the fabrication, characterization and applications of the ion-implanted waveguides in various optical glasses. The ion-implanted glass waveguides have shown good guiding performance as well as properties with respect to the bulks, suggesting promising potential for many aspects of photonics.
\end{abstract}

Keywords: Optical Glasses, Ion Implantation, Waveguide

\section{INTRODUCTION}

Optical waveguide is one of the basic components in integrated optics and optoelectronics (Townsend et al., 1994; Chen et al., 2007a; Nolte et al., 2003). It is defined as the high-refractive-index media surrounded by lowindex regions (Chen, 2009; Wang et al., 2009; Zhao et al., 2011). The guiding structure can confine light propagation in one Dimensional (1D) planar waveguide and in two Dimensional (2D) channel or ridge waveguide (Zhang et al., 2010). As the most fundamental and integral part of integrated optics circuits, the optical waveguide is of great importance in modern optical communication. Moreover, some properties of substrate materials in waveguide configurations can be considerably improved relative to those of the corresponding bulk materials. For example, compared with the substrate materials, waveguide structures can confine the light propagation within small volumes of the order of several microns, by which the optical density can reach very high levels even at lowpower pumpings, often resulting in a lower threshold and a higher gain (Chen et al., 2009; Vezenov et al., 2005). In addition, if the physical shapes and correlated modal profiles have suitable dimensions, $2 \mathrm{D}$ waveguides can be easily connected with optical fibers with a high coupling efficiency for construction of integrated photonic systems (Murphy, 1999).

Common requirements on material for integrated optics are as follows. (i) The materials can be utilized to fabricate high-quality optical waveguides. (ii) On the same substrate material, as many components with different features as possible can be produced. (iii) The cost of both the material and its corresponding processing is low, which largely determines the usefulness of integrated optical devices. Optical glasses, which can well meet these needs, are desirable materials for the fabrication of compact waveguide devices.

(C) 2013 Chun-Xiao Liu, Bo Peng, Wei Wei, Wei-Nan Li, Hai-Tao Guo and Shu Cheng. This open access article is distributed under a Creative Commons Attribution (CC-BY) 3.0 license which permits unrestricted use, distribution, and reproduction in any medium, provided the original work is properly credited. DOI: 10.3844/pisp.2013.1.12 
Compared with the optical crystals and semiconductors and polymers, optical glass waveguides have outstanding advantages, especially in the economic considerations.

Optical waveguides have been fabricated in various glass substrates using various techniques such as ion exchange (Veasey et al., 1999; Righini et al., 2001; Svecova et al., 2008), femtosecond laser inscription (Psaila et al., 2007; Valle et al., 2005; Mairaj et al., 2002), sol-gel (Najafi et al., 1998) and ion implantation (Okur and Townsend, 1997; Sum et al., 2005). Among these techniques, ion implantation possesses one of the most advantageous characteristics, that is, the wide applicability of materials (Chen et al., 2007a). It has been applied to produce versatile guiding devices in more than 100 materials, since the first proton-implanted waveguide in fused silica was reported in 1968 (Townsend et al., 1994). Moreover, ion implantation allows accurate control of both dopant composition and penetration depth through the choice of the species and the energy of the ions (Jaque and Chen, 2009; Kip et al., 1995).

Townsend et al. (1994) presented the first overview of the ion-implanted waveguides in insulating materials. Chen et al. (2007a) summarized the progress of the same research topics between 1994 to 2006. Chen (2008) reviewed the development of $2 \mathrm{D}$ waveguide production in insulating materials by ion implantation. However, owing to the research field is highly active, there have been in recent years a large number of achievements in this area that make necessary and updated review summarizing them. In this work, we have focused on photonic guing structures in optical glasses produced by ion implantation, giving a state-of-the-art summary of the field. The main content in the review is as follows. Section 2 described the basic methods used for the fabrication and characterization of ion-implanted waveguides. Section 3 summarized the recent results of optical waveguides in various optical glasses formed by ion implantation and their properties. Section 4 introduced the main applications related ion-implanted waveguides in optical area.

\subsection{Fabrication and Investigation Methods}

We will describe the fabrication and investigation methods of ion-implanted waveguides. For details, some important aspects of ion implantation such as mechanisms and processing, refractive index reconstruction method (reflectivity calculation method), waveguide coupling techniques (prism-coupling and end-face coupling methods), annealing treatments and loss measurement methods will be discussed.

\subsection{Ion Implantation Technique}

Ion implantation is one of the most important techniques for modifying materials surface properties and it has been proven to be an effective method to fabricate waveguides, owing to its superior controllability and reproducibility. Compared with other techniques such as ion exchange and metal ion in diffusion, ion implantation is not limited to the Curie temperature of substrate materials. In the research area of ion-implanted waveguides, accelerators are more often used because they offer high energies of specific implanted ions at acceptable doses. The implanted ions, normally with positive charges, are extracted out from the sources, experiencing acceleration and mass/energy selection and bombarded into the target materials by beam scanning technique, by which uniform irradiation is ensured over the sample surface (Chen et al., 2007a). The ion implanted waveguide technique is sorted to two different strategies named light-ion implantation and heavy-ion implantation, according to the mass of implanted ions. The ions used in the light-ion implantation are protons or Helium ions, however, the ions used in the heavy one usually are $\mathrm{C}^{+}, \mathrm{O}^{+}$and $\mathrm{Cu}^{+}$. The light-ion implantation requires higher dose than the heavy-ion implantation to form waveguide structures. In some materials, fluence of only $10^{13}$ ions $/ \mathrm{cm}^{2}$ magnitude is sufficient for the heavy-ion implanted waveguide formation.

During implantation processes, the losses of incident ions include two different kinds of physical mechanisms called nuclear energy deposition and electronic energy deposition. Those energy depositions would cause the distortion of the substrate material near the surface region and induce the variation of refractive index. At the end of the ion range, an optical barrier with a relatively low refractive index compared with the substrate is formed by the nuclear energy deposition. Meanwhile, refractive index would increase or be slightly disturbed between the air and the optical barrier, which is mainly decided by electronic energy deposition. The propagation light would be well confined by air and optical barrier in optical waveguide. Therefore, we can control the ion species, energies, doses and post-annealing treatments to fabricate valuable waveguides.

The 1D planar waveguide could be fabricated by direct ion implantation, since it only needs entire implantation of the bulk surface. For 2D channel waveguides, we can make use of mask-assisted ionimplantation to form surface structures. Not to lose the generality, helium-implanted $\mathrm{Nd}: \mathrm{CBN}$ optical-stripe waveguide is discussed here as an example. 


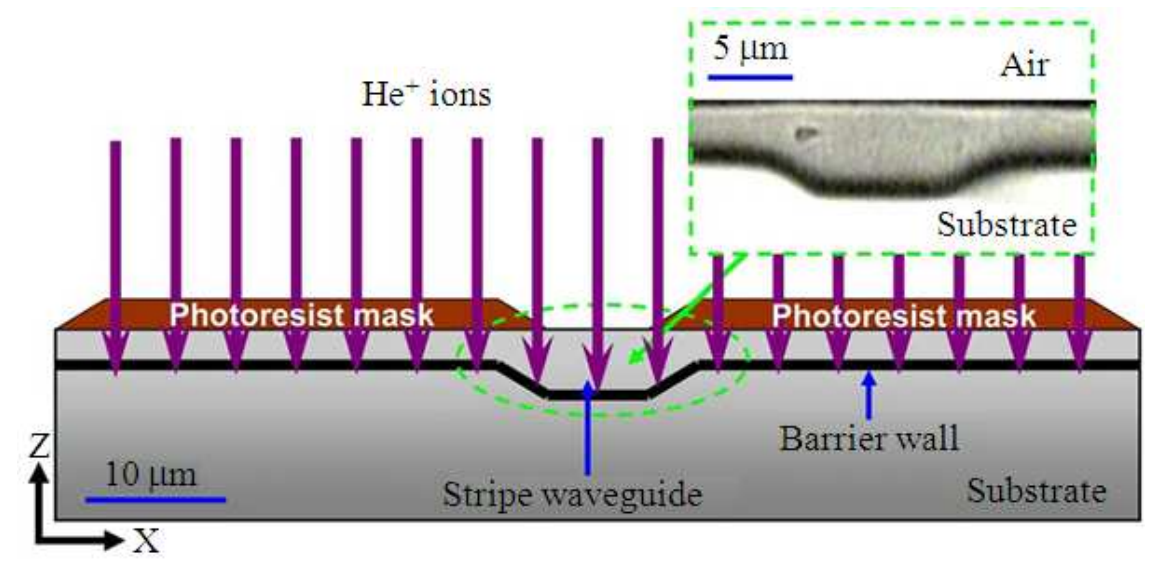

Fig. 1. (Color online) Schematic of the stripe waveguide fabrication process in a Nd:CBN crystal. The inset shows the microscope image of the stripe waveguide cross section (data obtained from Tan et al., 2009)

Figure 1 shows the schematic plot of the mask-assistant ion implantation for the channel waveguide fabrication in neodymium-doped calcium barium niobate crystals. In the process, triple-energy helium ions are implanted into the sample surface containing a series of straight photoresist stripes. Owing to the blocking effect of the photoresist mask stripes, the incident He ions construct a ridged barrier wall buried inside the substrate, forming stripe waveguides in $\mathrm{Nd}: \mathrm{CBN}$ (Tan et al., 2009). In addition, if the implantation induces index increases of irradiated regions, channel waveguides can be fabricated by one-step ion implantation with standard lithograph in some materials, such as $\mathrm{LiNbO}_{3}$ (Chen et al., 2007b).

\subsection{Thermal Treatment}

High energy ion implantation can form waveguide in many optical materials, but meanwhile produces point defects or color centers by electronic excitation and nuclear collisions, which will unavoidably increase propagation loss in the waveguide due to absorption or scattering effects. Thermal annealing is a means of altering the residual effects of densification, viscous flow and anisotropic deformation in the implanted layer, improving the electro-optic or non-linear optic properties (Johnson et al., 1996). Conventional furnace annealing (Gumennik et al., 2005), rapid thermal annealing (Feuster et al., 1994) and laser annealing (Townsend and Olivares, 1997) are most commonly used to improve the optical performance of all the ion-implanted waveguides fabricated by the technique of ion implantation, reducing both the frequency of color centers and propagation loss. In the case of conventional furnace annealing treatment, samples are annealed for a few tens of minutes to several hours at temperatures ranging from $200-500^{\circ} \mathrm{C}$ in some atmosphere (air, nitrogen and so on) or vacuum conditions, depending on the substrate properties. Moreover, the rapid thermal annealing can reduce the induced defects and avoid unwanted diffusion of the implanted ions at the same time. For laser annealing, laser energy may be confined to the surface layer by using strongly absorbed radiation, resulting in local heating which can remove intrinsic defects and induce crystalline regrowth or solid phase epitaxy.

\subsection{Waveguide Coupling Methods}

The basic investigations of waveguides are to evaluate the confinement of light and measure the effective refractive index of the guided modes. The corresponding methods are end-face coupling and prism coupling, respectively.

\subsection{Prism Coupling Method}

The well-known prism-coupling method is used to measure the effective refractive indices of the guided modes. During the prism-coupling measurement, a laser beam strikes the base of a rutile prism, which is brought into contact with the samples. A silicon photo detector is used to detect the intensity of the reflected light from the prism bottom. The prism, waveguide and photo detector are mounted on a rotary table so that the incident angle of the laser beam could be adjusted. When the resonant condition is satisfied, the laser beam would be coupled into the waveguide region and a lack of reflected light would result in a dip. Combining with the prism refractive index and the dark-mode spectra, the effective refractive indices of the guided modes could be calculated. 


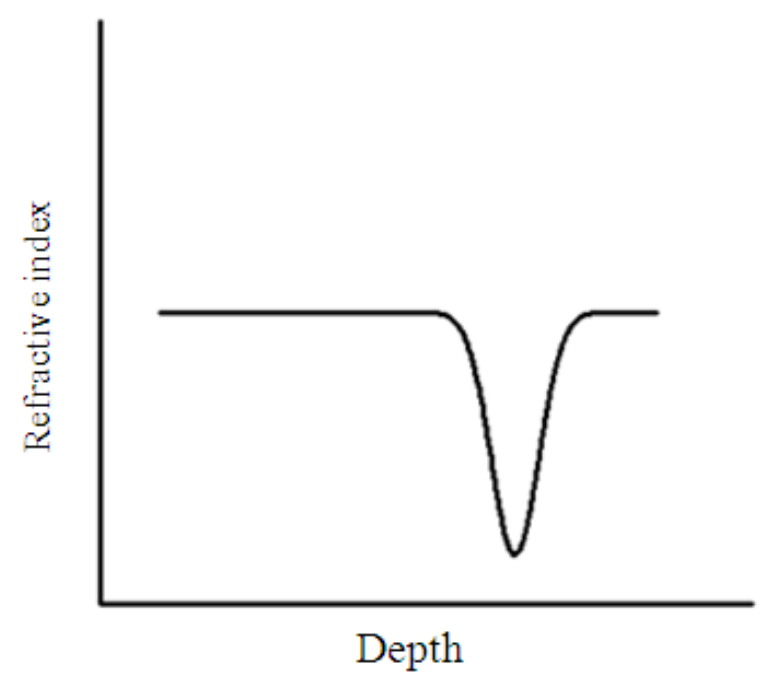

(a)

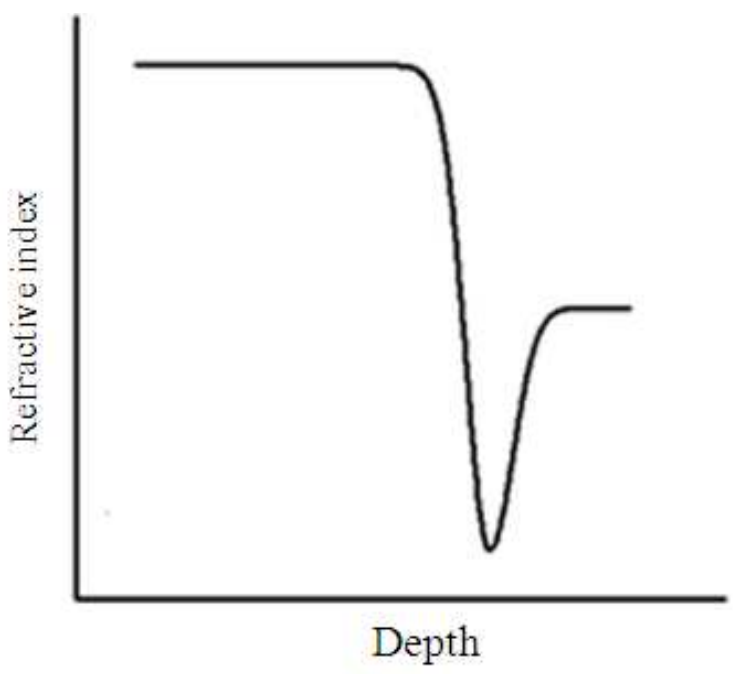

(b)

Fig. 2. (Color online) Schematics of the refractive-index profiles of the ion-implanted waveguides: (a) barrier-type and (b) enhanced-well + barrier-type

\subsection{End-Face Coupling Method}

A conventional end-face coupling optical system is carried out to record the near-field intensity distribution. During the measurement, a light beam, either focused by microscopic lenses or directly output from a fiber, is coupled into the waveguide to excite the guided modes and another microscope objective lens collects the light from the output facet of the waveguide, which is imaged onto a CCD camera.

\subsection{Refractive Index Distribution}

Optical waveguides are defined as high-refractiveindex material surrounded by low-index layer. The refractive index distribution is very important for investigating the characteristics of optical waveguides. It decides the waveguide properties such as guide mode and the nonlinear properties. To study the properties of waveguides, the refractive index distribution would be a basic problem. The related investigation will provide theory instruction for optical waveguide devices. Owing to the direct measurement of the refractive index distribution is quite complicated, many methods have been developed to reconstruct the refractive index distribution, including the inverse Wentzel-KramersBrillouin (White and Heidrich, 1976), the parameterized index profile reconstruction (Fluck et al., 1993) and the Reflectivity Calculation Method (RCM) (Chandler and Lama, 1986). The RCM based on the effective refractive indices of the guided modes has been proven to be particularly successful for ion-implanted waveguides. In this method, we firstly choose a conceivable function for the index profile characterized by a set of parameters, which is constructed from two half Gaussians. secondly, the mode indices of this hypothetical profile are calculated by RCM. Thirdly, we compare these theoretical mode index values with the experimental ones obtained from dark-mode spectroscopy (so-called m-lines). Lastly, keep changing the parameters to alter the index profile shape until the theoretical mode indices match the experimental ones within a satisfactory error. The final profile is therefore assumed to approximately depict the refractive index distribution in the waveguide.

As shown in Fig. 2, refractive index distributions of ion-implanted waveguides usually are divided into two categories: barrier-type and enhanced-well+ barrier-type index profiles. The well-known distribution is the "optical-barrier"-confined one, which was first proposed by Townsend et al. (1994). The optical barrier could be the result of the damage suffered as a consequence of the nuclear stopping of the implanted ions at the end of the tracks. The light can be confined between the barrier and the sample surface (air). The typical "enhanced-well+ barrier-type" distribution contains an enhanced well with a positive index change in the waveguide region and an optical barrier with a negative index change at the boundaries of the waveguide and the substrate. This means the waveguide is confined not only by the indexenhanced well but also by the optical barrier. 


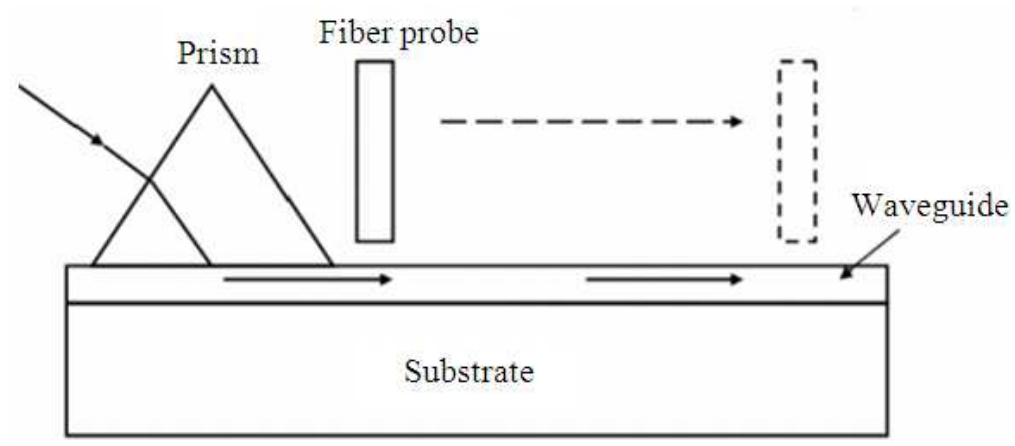

Fig. 3. Schematic of the set-up for the moving fiber probe technique (data obtained from Li et al., 2005)

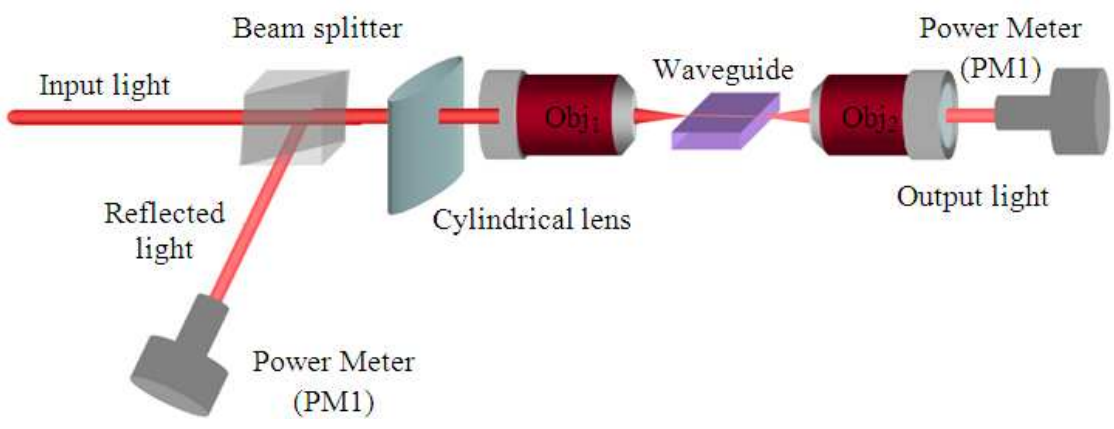

Fig. 4. Schematic of the experimental setup for loss measurement through back-reflection method

\subsection{Waveguide Loss Measurement}

The propagation loss is one of the most critical parameters for evaluating the guiding properties of waveguides. It could be measured based on the coupling techniques mentioned earlier. The attenuation of ionimplanted waveguides is mainly caused by absorption, surface scattering, refractive index profiles and end facet polishing quality. A number of methods have been developed to measure the waveguide propagation loss, including moving fiber probe techniques (Wang et al., 2007), back-reflection methods (Ramponi et al., 2002) and Fabry-Perot resonance methods (Regener and Sohler, 1985).

\subsection{Moving Fiber Probe Technique}

The moving fiber probe technique bases on the prism-coupling system. The schematic set-up is shown in Fig. 3. When a laser beam excites a particular waveguide mode, a fiber probe scans down the length of the propagation streak to measure the scattered light from the surface of the waveguide. The detected light intensity is plotted as a function of the propagation distance. A computer code based on least-squares theory is used to compute the measured loss.

\subsection{Back-Reflection Method}

The back-reflection method is suitable for measuring the propagation loss of planar waveguides, as shown in Fig. 4. In this method, the injected light is divided into two beams by a beam splitter. One of them is monitored by a detector (PM1). A cylindrical lens couples the light into the waveguide. A microscope objective lens couples the light out of the waveguide and images the near-field mode profile onto another detector (PM2). The sample is mounted on a six-dimensional stage to achieve the maximum coupling efficiency between the injected light and the fundamental mode of the planar waveguide. According to the given formula (Ramponi et al., 2002) and the measured parameters, the propagation loss can be calculated.

\subsection{Fabry-Perot Resonance Method}

In Fig. 5, the laser beam is end-face coupled to the channel waveguide resonator, which is placed in a small crystal oven. The near-field intensity distribution of the transmitted light is recorded by a detector. 


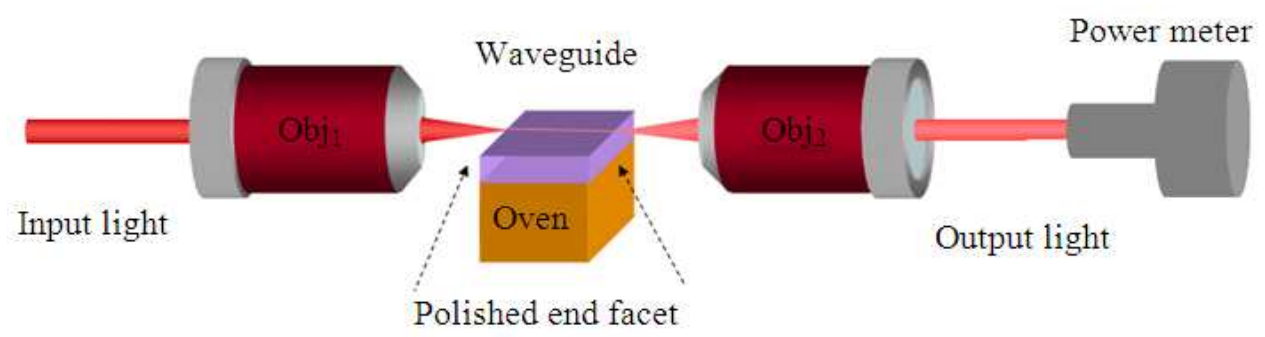

Fig. 5. Schematic of the experimental setup for loss measurement through Fabry-Perot resonance method

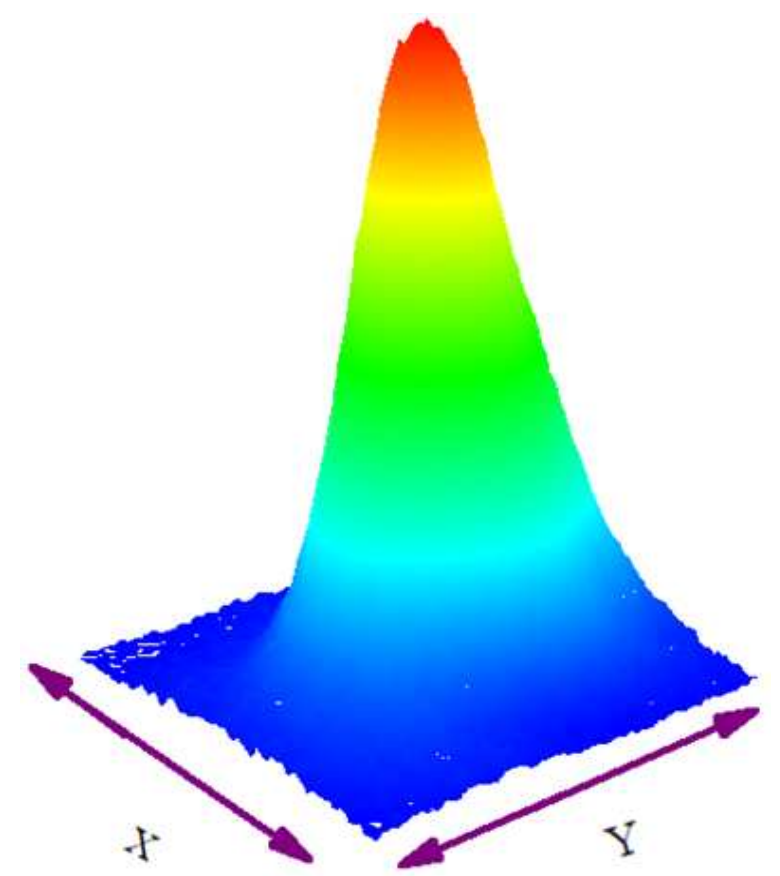

Fig. 6. Near-field intensity distribution of quazi- $\mathrm{TE}_{00}$ mode of the channel waveguide in $\mathrm{Yb}^{3+}$-doped silicate glasses (data obtained from Liu et al., 2012a)

Simultaneously, a fraction of the transmitted intensity could be measured by a photodiode as a function of the crystal temperature (Regener and Sohler, 1985). Based on the experimental results and the standard formula, the channel waveguide propagation loss can be evaluated.

\subsection{Optical Glasses}

Various optical glasses including silicates, phosphates, tellurites and chalcogenides are perfect hosts for doping of various rare earth ions, such as $\mathrm{Yb}^{3+}, \mathrm{Er}^{3+}$ and $\mathrm{Nd}^{3+}$, showing excellent laser performances and practical applications in optical communication. The most common method for waveguide formation in optical glasses is ion exchange, in which waveguides are fabricated by the exchange of specific ions between the substrate and the ambient chemical liquid (Chen, 2008; Ramaswamy and Srivastava, 1988). However, ion implantation has been successfully utilized to manufacture guiding structures in a wide range of optical glasses.

\subsection{Silicate Glass}

Due to the high solubility for rare-earth ions and good chemical stability, silicate glasses are excellent candidates for waveguide amplifiers and lasers. Planar and channel waveguides in silicate glasses have been fabricated by the conventional exchange of $\mathrm{K}^{+} / \mathrm{Na}^{+}$or $\mathrm{Ag}^{+} / \mathrm{Na}^{+}$ions.

Kakarantzas et al. (2003) reported the fabrication of standard optical barrier-type waveguides in silicate glasses by implanting helium ions at a fluence of $\sim 10^{16}$ ions $/ \mathrm{cm}^{2}$ in 1992 . In this case, the low-index barrier is the only confinement for the waveguide, which degrades the waveguide quality (Townsend et al., 1994). In detail, the longitudinal width of the barrier normally is small, which results in the obvious leakage of the light energy through the barrier into the substrate regions due to optical tunneling effects. This phenomenon is obvious for all barrier-confined waveguides, particularly when the barrier layers are very thin (Chen et al., 2007a). Nevertheless, multiple-energy ion implantations are necessary to solve the problem, which can broaden the barrier. Therefore, the tunneling effect can be considerably reduced, resulting in a better confinement of the light modes. Liu et al. (2012a) have obtained monomode optical planar and channel waveguides in $\mathrm{Yb}^{3+}$-doped silicate glasses by $(450+500+550) \mathrm{keV}$ helium ion implantation at the doses of $(2+2+2) \times 10^{16}$ ions $/ \mathrm{cm}^{2}$. After the triple-energy $\mathrm{He}^{+}$implantation, the optical barrier is broadened to $0.2 \mu \mathrm{m}$, which improves the guiding properties. Figure 6 shows the threeDimensional (3D) near-field intensity distribution of the $\mathrm{TE}_{00}$ mode of the channel waveguide. As one can see, the light is indeed confined both in the vertical and in the 
lateral direction. On the other hand, the refractive index of waveguides, especially for the heavy ion-implanted cases, might first experience a positive change in the main region of the incident ion's trajectory, forming an enhanced index well, which confines the guided light in a non-leaky manner. Wang et al. (2007) have reported on the fabrication and characterization of low-loss planar and stripe waveguides in a $\mathrm{Nd}^{3+}$-doped glass by $6.0 \mathrm{MeV}$ oxygen-ion implantation at a dose of $1 \times 10^{15}$ ions $/ \mathrm{cm}^{2}$. The planar waveguides in the $\mathrm{Yb}^{3+}$-doped silicate glasses have also been fabricated by $4.0 \mathrm{MeV} \mathrm{C}^{2+}$ and $6.0 \mathrm{MeV}$ $\mathrm{O}^{3+}$ ion implantation, respectively (Liu et al., 2012b; 2012c). Recently, Liu et al. (2013) have also reported the low-dose carbon-implanted planar waveguides in $\mathrm{Er}^{3+} / \mathrm{Yb}^{3+}$ co-doped silicate glasses. All of the above results show that a refractive index enhanced region as well as an optical barrier have been created after the heavy ion-implantation processing.

Owing to the complex compositions and disordered structures, the mechanism of waveguide formation in silicate glasses is usually complicated. The positive change of refractive index in the waveguide region may be due to the compaction effect during the process of implantation, which is usually accompanied by an increase in physical density in the corresponding region (Townsend et al., 1994; Chen et al., 2007a; Wang et al., 2007). Moreover, Liu et al. (2012b; 2012c) have thought, the non-bridging oxygens induced by the implantationirradiation in the waveguide region may also cause the formation of the enhanced well, which is due to the fact that the ionic refractivity of non-bridging oxygens is larger than that of bridging oxygens in the oxide glasses. However, it should be noted that no positive index change was observed in Kakarantzas et al. (2003) work. Further experimental investigations are required for greater understanding. The optical barrier could be the result of damage suffered as a consequence of the nuclear stopping of the implanted ions at the end of the tracks, where decreases in both physical density and refractive index. The view on the optical barrier has been accepted by more and more researchers (Townsend et al., 1994; Chen et al., 2007a; Wang et al., 2007; Jiang et al., 2000). Liu et al. (2012d) have recently reported the characterization of planar waveguides in $\mathrm{Yb}^{3+}$-doped silicate glasses fabricated by double-energy proton implantation at a total dose of $3 \times 10^{16}$ ions $/ \mathrm{cm}^{2}$ and pointed out implanting ions how to get along with the components of the substrate. The work introduces the ion exchange between proton and $\mathrm{Na}^{+}$ions. As a result, $\mathrm{Na}^{+}$ ion is replaced by $\mathrm{H}^{+}$ion during the reaction of ion exchange and then occupies the void that was caused by proton implantation at the end of ions track or migrates from the vicinity of the damage peak. As one know, the radius of $\mathrm{H}^{+}$ion is much smaller than that of $\mathrm{Na}^{+}$ion, so the structure of the silicate glass becomes relaxed and the volume turns large in the terminal region. Therefore, both the physical density and the refractive index reduce reasonably and the optical barrier is formed.

\subsection{Phosphate Glass}

Owing to the rapid development of both optical communication and series high power laser devices used as the laser driven Inertia Confined Fusion (ICF), phosphate glasses, especially $\mathrm{Er}^{3+} / \mathrm{Yb}^{3+}, \mathrm{Nd}^{3+}$ and $\mathrm{Yb}^{3+}$ doped phosphate glasses have received more and more attentions. Among a variety of $\mathrm{Er}^{3+}$-doped materials, phosphate glass has been proven to be one of the most promising hosts for the high concentration doping of $\mathrm{Er}^{3+}$ ions. $\mathrm{Nd}^{3+}$-doped phosphate glass is one of the excellent laser materials and has been extensively used for the laser output at $1.064 \mu \mathrm{m}$. $\mathrm{Yb}^{3+}$-doped phosphate glasses exhibit high emission cross-section, broad absorption and emission band and long fluorescence lifetime (1-2 $\mathrm{ms})$, making them particularly attractive to generate ultra short pulses and tunable laser sources. The most common method for waveguide fabrication in phosphate glasses is the process of ion exchange, where alkali ions in the glass are exchanged for ions with larger polarizability, thus causing an increase in the refractive index near the glass surface (Veasey et al., 2000). Alternatively, ion implantation has become one of the promising techniques to produce waveguides in phosphate glasses.

Earlier work on the ion-implanted waveguides in phosphate glasses was done by Chen et al. (2002). They reported the planar waveguides formation in $\mathrm{Er}^{3+} / \mathrm{Yb}^{3+}$ co-doped phosphate glasses by the implantations of $\mathrm{He}^{+}$ or $\mathrm{Si}^{+}$ions, respectively. It was found that the refractive indices in the waveguide and the barrier regions have considerable negative changes after the ion implantations. By combining $\mathrm{He}^{+}$ion implantation and $\mathrm{Ar}^{+}$sputtering etching, Tan et al. (2007) fabricated ridge waveguides in $\mathrm{Er}^{3+} / \mathrm{Yb}^{3+}$ co-doped phosphate glasses. Its optical characterization showed that there is little refractive index change in the guiding region and a barrier with a lower refractive index at the end of ion range. Recently, Liu et al. $(2011 ; 2012$ e) investigated the optical properties of helium-implanted waveguides in $\mathrm{Nd}^{3+}$ and $\mathrm{Yb}^{3+}$-doped phosphate glasses, respectively. In the study, the refractive indices in the waveguide and the barrier regions were reduced by 0.005 and 0.119 in $\mathrm{Nd}^{3+}$ -doped phosphate glasses, while in $\mathrm{Yb}^{3+}$-doped 
phosphate glasses, these index decrease in the two regions were 0.007 and 0.050 , respectively.

As one can see, all above-mentioned results show the ion-implanted phosphate glass waveguides were typical barrier-confined. The propagation losses of such waveguides usually are high, because of the relatively poor confinement of the light inside the waveguide region. In order to obtain acceptable guiding properties of the waveguides in $\mathrm{Nd}^{3+}$ and $\mathrm{Yb}^{3+}$-doped phosphate glasses formed by triple-energy helium implantation, Liu et al. (2011; 2012e) carried out continuous annealing treatments in atmosphere. They found that the refractive indices in the waveguide regions, to varying degrees, rise with each annealing treatment, owing to recovery of damages. Furthermore, the attenuation values of helium-implanted $\mathrm{Nd}^{3+}$ and $\mathrm{Yb}^{3+}$-doped phosphate glass waveguides could be considerably reduced to be $\sim 2.06$ and $1.52 \mathrm{~dB} / \mathrm{cm}$ after the thermal treatments, respectively.

\subsection{Fused Silica Glass}

Fused silica (quartz) is a high purity synthetic amorphous silicon dioxide with only two elements $\left(\mathrm{SiO}_{2}\right)$. It has a number of excellent properties that include near zero thermal expansion, excellent transparency from the UV to the near IR, good chemical inertness, low dielectric constant and low cost. Moreover, the refractive index of fused silica is very close to that of the commercial fibers, making it a attractive substrate material for integrated optics applications (Harry et al., 2006).

Schineller et al. (1968) reported the first ionimplanted planar waveguides in fused silica by using proton implantation. By combining $\mathrm{B}$ or $\mathrm{Li}$ ion implantation and lithographic technique, Kersten et al. (1991) studied the first ion-implanted channel waveguides in 1976. Since then, numerous researchers have investigated the waveguides formed in fused silica and analyzed their properties. Two representative examples are as following. Wang et al. (2004) reported on planar waveguides in fused silica by $3.0 \mathrm{MeV}$ oxygen ion implantation at the dose of $1 \times 10^{15}$ ions $/ \mathrm{cm}^{2}$ and attenuation coefficient of $0.14 \mathrm{~dB} / \mathrm{cm}$ was obtained for the thermal treated samples. Leech et al. (1996) investigated channel waveguides in fused silica produced by $\mathrm{MeV} \mathrm{Si}, \mathrm{P}$ or $\mathrm{Ge}$ ion implantation, in which the propagation losses were only of 0.1 to $0.2 \mathrm{~dB} / \mathrm{cm}$ at the telecommunication wavelength of 1.3 and $1.5 \mu \mathrm{m}$. As mentioned above, one of most important characteristics for ion-implanted fused silica waveguides is low loss. Previous studies have shown that the waveguide with optical losses of less than $1 \mathrm{~dB} / \mathrm{cm}$ can be used in integrated optics. Therefore, ion implantation is a practical method for effective waveguide formation in fused silica comparable with other techniques. Owing to the compaction under the influence of the nuclear and electronic damage, the refractive index of the fused silica has a positive change to some extent, forming index-enhanced wells, which means fine confinement of the light inside the waveguides and hence low propagation losses.

\subsection{Tellurite Glass}

Tellurite glass is proposed as a promising candidate for the fabrication of active integrated optical circuits because of their high rare-earth solubility and large stimulated emission cross sections (Zhou et al., 2010). Especially, $\mathrm{Er}^{3+}$-doped tellurite glass is suitable for gainflattened optical amplifiers in Wavelength-Division Multiplexer (WDM) applications due to their broad emission bandwidth around the 1.55 micron wavelength and low phonon energy.

Berneschi et al. (2007) reported the formation of channel waveguide in $\mathrm{Er}^{3+}$-doped tellurite glasses by 1.5 $\mathrm{MeV} \mathrm{N} \mathrm{N}^{+}$ion implantation at dose of $1 \times 10^{16}$ ions $/ \mathrm{cm}^{2}$, with a $75 \mu \mathrm{m}$ thick and $24 \mu \mathrm{m}$ gap silicon stripe mask. Since the nitrogen ion implantation induced positive refractive index changes, one-step implantation with a rectangular mask can produce the channel waveguide. The 2D stripe waveguide in $\mathrm{Er}^{3+}$-doped tellurite glasses was also fabricated by Khanh et al. (2009). It was found that the implanted layer exhibited a decrease of the refractive index with respect to the virgin bulk glass and a barrier with a lower refractive index was built up at the end of the ion track. Therefore, the light can be confined in vertical direction. Moreover, with the blocking effect of the photoresist stripes at the wedged edges, the incident nitrogen ions experience different penetration depth inside the $\mathrm{Er}^{3+}$-doped tellurite glass, creating semiclosed barrier walls with trapezoidal-shaped sections. These special barrier walls constructed a low refractive index well, which confines the light in horizontal direction around the implanted region (Chen, 2008).

\subsection{Other Glasses}

The ion-implanted waveguides were also fabricated in some other glasses, such as germanate, filter and chalcogenide glasses. Shepherd et al. (1994) reported on the first Tm-doped germanate glass waveguides formed by $\mathrm{He}$ et al. (1998) implantation at energy of $2.9 \mathrm{MeV}$ and dose of $1 \times 10^{16}$ ions $/ \mathrm{cm}^{2}$ at liquid-nitrogen temperature. $\mathrm{He}^{+}$and $\mathrm{H}^{+}$ions at different energies and 
doses were used to make optical waveguides in filter glasses by Okur and Townsend (1997). Recently, Qiu and Narusawa (2010) have reported on the fabrication of planar optical waveguides in gallium lanthanum sulphide glasses by $350 \mathrm{keV}$ proton implantation at a fluence of $1 \times 10^{15}$ ions $/ \mathrm{cm}^{2}$.

\subsection{Applications}

Ion-implanted glass waveguides have been already used for applications, including second harmonic generation, waveguide amplifiers and waveguide lasers. Since the nonlinear susceptibility is preserved or only slightly reduced in the ion-implanted waveguide, ion implantation possibly is one of the most effective methods to obtain optical waveguides with applications in Second Harmonic Generation (SHG). Babsail et al. (1991) reported on the second-harmonic generation in ion-implanted quartz planar waveguides. In recent years Er-Doped Waveguide Amplifiers (EDWA) have attracted particular attention for their wide applications in telecommunication for compensation of optical signal losses at $\sim 1.5 \mu \mathrm{m}$. Liu et al. (2004) produced EDWA in $\mathrm{Er}^{3+} / \mathrm{Yb}^{3+}$ co-doped phosphate glasses through focused proton beam writing with energy of $2.0 \mathrm{MeV}$ and doses of $1 \times 10^{14}-1 \times 10^{15} \mathrm{ions} / \mathrm{cm}^{2}$. The results showed that the maximum net gain of the waveguide amplifiers at $1.534 \mu \mathrm{m}$ wavelength was measured to be $\sim 1.72 \mathrm{~dB} / \mathrm{cm}$ with $100 \mathrm{~mW}$ pump power at $975 \mathrm{~nm}$ wavelength. Waveguide lasers often show low pumping thresholds and enhanced efficiencies with respect to bulk lasers, due to the optical intensities of the waveguide layers are higher than that of the bulk. Shepherd et al. (1994) demonstrated $1.9-\mu \mathrm{m}$ waveguide laser in Tm: lead germanate glasses fabricated by helium-ion implantation.

\section{CONCLUSION}

In this review we have focused on the development of ion-implanted waveguides in optical glasses, including the fabrication methods, properties and applications. With combination of photolithography technique, channel waveguides could be fabricated by ion implantation. The optimization of the waveguide parameters can be achieved by suitable annealing treatments. Based on ion-implanted glass waveguides, second harmonic generation, waveguide amplifiers and waveguide lasers have been realized for practical applications.

\section{ACKNOWLEDGEMENT}

This study was financially supported by the National Natural Science Foundation of China (Grant Nos. 61077070, 10876009, 51002181 and 61177084) and the
Open Research Fund of State Key Laboratory of Transient Optics and Photonics, Chinese Academy of Sciences.

\section{REFERENCES}

Babsail, L., G. Lifante and P.D. Townsend, 1991. Second-harmonic generation in ion-implanted quartz planar waveguides. Applied Phys. Lett., 59: 384386. DOI: 10.1063/1.105466

Berneschi, S., G.N. Conti, I. Banyasz, A. Watterich and N.Q. Khanh et al., 2007. Ion beam irradiated channel waveguides in $\mathrm{Er}^{3+}$-doped tellurite glass. Applied Phys. Lett., 90: 121136-121138. DOI: 10.1063/1.2717085

Chandler, P.J. and F.L. Lama, 1986. A new approach to the determination of planar waveguide profiles by means of a non-stationary mode index calculation. Opt. Acta: Int. J. Opt., 33: 127-143. DOI: 10.1080/713821921

Chen, F., 2008. Construction of two-dimensional waveguides in insulating optical materials by means of ion beam implantation for photonic applications: Fabrication methods and research progress. Crit. Rev. Solid State Mater. Sci., 33: 165-182. DOI: 10.1080/10408430802310868

Chen, F., 2009. Photonic guiding structures in lithium niobate crystals produced by energetic ion beams. J. Applied Phys., 106: 081101-081129. DOI: 10.1063/1.3216517

Chen, F., X.L. Wang and K.M. Wang, 2007a. Development of ion-implanted optical waveguides in optical materials: A review. Opt. Mater., 29: 15231542. DOI: 10.1016/j.optmat.2006.08.001

Chen, F., X.L. Wang, X.S. Li, L.L. Hu and Q.M. Lu et al., 2002. Ion-implanted waveguides in $\mathrm{Nd}^{3+}$-doped silicate glass and $\mathrm{Er}^{3+} / \mathrm{Yb}^{3+}$ co-doped phosphate glass. Applied Surf. Sci., 193: 92-101. DOI: 10.1016/S0169-4332(02)00212-X

Chen, F., Y. Tan and D. Jaque, 2009. Ion-implanted optical channel waveguides in neodymium-doped yttrium aluminum garnet transparent ceramics for integrated laser generation. Opt. Lett., 34: 28-30. DOI: 10.1364/OL.34.000028

Chen, F., Y. Tan, L. Wang, Q.M. Lu and H.J. Ma, 2007b. Oxygen ion-implanted optical channel waveguides in $\mathrm{Nd}: \mathrm{MgO}: \mathrm{LiNbO}_{3}$ : Fabrication, characterization and simulation. J. Phys. D: Applied Phys., 40: 58245827. DOI: 10.1088/0022-3727/40/19/004 
Feuster, M., C. Buchal, E. Sneoks and A. Polman, 1994. Rapid thermal annealing of $\mathrm{MeV}$ erbium implanted $\mathrm{LiNbO}_{3}$ single crystals for optical doping. Applied Phys. Lett., 65: 225-227. DOI: 10.1063/1.112680

Fluck, D., D.H. Jundt, P. Gunter, M. Fleuster and C. Buchal, 1993. Modeling of refractive index profiles of $\mathrm{He}^{+}$ion-implanted $\mathrm{KNbO}_{3}$ waveguides based on the irradiation parameters. J. Applied Phys., 74: 6023-6031. DOI: 10.1063/1.355217

Gumennik, A., A.J. Agranat, I. Shachar and M. Hass, 2005. Thermal stability of a slab waveguide implemented by $\alpha$ particles implantation in potassium lithium tantalate niobate. Applied Phys. Lett., 87: 251917-251919. DOI: 10.1063/1.2147723

Harry, G., T. Corbitt, M. Freytsis, D. Ottaway and N. Mavalvala et al., 2006. Mechanical loss of laserwelded fused silica fibers. Rev. Sci. Instrum., 77: 023906-023908. DOI: 10.1063/1.2170075

He, B., B. Yang, J. Lundahl, S. Fredrikson and J. Hillert, 1998. The myelin basic protein gene in multiple sclerosis: Identification of discrete alleles of a $1.3 \mathrm{~kb}$ tetranucleotide repeat sequence. Acta Neurol. Scand. 97: 46-51. PMID: 9482678

Jaque, D. and F. Chen, 2009. High resolution fluorescence imaging of damage regions in $\mathrm{H}+$ ion implanted Nd:MgO: $\mathrm{LiNbO}_{3}$ channel waveguides. Applied Phys. Lett., 94: 011109-011111. DOI: 10.1063/1.3064164

Jiang, S., T. Luo, B.C. Hwang, F. Smekatala and K. Seneschal et al., 2000. $\mathrm{Er}^{3+}$-doped phosphate glasses for fiber amplifiers with high gain per unit length. J. Non-Cryst. Solids, 263-264: 364-368. DOI: 10.1016/S0022-3093(99)00646-8

Johnson, C.M., M.C. Ridgsway and P.W. Leech, 1996. Thermal annealing of implantation-induced compaction for improved silica waveguide performance. Applied Phys. Lett., 69: 984-986. DOI: 10.1063/1.117103

Kakarantzas, G., A. Ortigosa-Blanch, T.A. Birks, P.S.J. Russell and L. Farr et al., 2003. Structural rocking filters in highly birefringent photonic crystal fiber. Opt. Lett., 28: 158-160. DOI: 10.1364/OL.28.000158

Kersten, D.J., P. Mamassian and D. Knill, 1991. Moving cast shadows generate illusory object trajectories. Investig. Ophthalmol. Visual Sci., 32: 1179-1179.

Khanh, N.Q., S. Berneschi, I. Banyasz, M. Brenci and M. Fried et al., 2009. Fabrication of channel waveguides in $\mathrm{Er}^{3+}$-doped tellurite glass via $\mathrm{N}^{+}$ion implantation. Nucl. Instrum. Methods B, 267: 23272330. DOI: $10.1016 /$ j.nimb.2009.03.042
Kip, D., S. Aulkemeyer and P. Moretti, 1995. Low-loss planar optical waveguides in strontium barium niobate crystals formed by ion-beam implantation. Opt. Lett., 20: 1256-1258. DOI: 10.1364/OL.20.001256

Leech, P.W., M.C. Ridgway and M.F. Faith, 1996. Channel waveguides formed in fused silica and silica-on-silicon by $\mathrm{Si}, \mathrm{P}$ and $\mathrm{Ge}$ ion implantation. IEE Proc. Optoelect., 143: 281-286. DOI: 10.1049/ip-opt:19960355

Li, S.L., K.M. Wang, F. Chen, X.L. Wang and G. Fu et al., 2005. Property studies of optical waveguide formed by $6.0 \mathrm{MeV}$ carbon ion implantation into Nd:silicate glass. J. Phys. D: Applied Phys., 38: 2899-2903. DOI: 10.1088/0022-3727/38/16/023

Liu, C.X., B. Peng, W. Wei, S. Cheng and W.N. Li et al., 2013. Low-dose carbon-implanted planar waveguides in $\mathrm{Er}^{3+} / \mathrm{Yb}^{3+}$ co-doped silicate glasses. Nucl. Instr. Methods Phys. Res. Sec. B: Beam Inter. Mater. Atoms, 295: 85-88. DOI: 10.1016/j.nimb.2012.11.010

Liu, C.X., S. Cheng, H.T. Guo, W.N. Li and W. Wei et al., 2012c. Optical properties of $\mathrm{Yb}^{3+}$-doped silicate glasses waveguides formed by low-dose oxygen ion implantation and effects of annealing. Optik-Int. J. Light Elect. Opt. DOI: 10.1016/j.ijleo.2012.05.019

Liu, C.X., S. Cheng, H.T. Guo, W.N. Li and X.H. Liu et al., 2012d. Proton-implanted optical planar waveguides in $\mathrm{Yb}^{3+}$-doped silicate glasses. Nucl. Instrum. Methods B, 289: 18-21. DOI: 10.1016/j.nimb.2012.08.004

Liu, C.X., S. Cheng, J.H. Zhao, W.N. Li and W. Wei et al., 2012a. Monomode optical planar and channel waveguides in $\mathrm{Yb}^{3+}$-doped silicate glasses formed by helium ion implantation. Opt. Laser Technol., 44: 2323-2326. DOI: 10.1016/j.optlastec.2012.04.033

Liu, C.X., S. Cheng, W.N. Li, W. Wei and B. Peng, 2012b. Monomode optical waveguides in $\mathrm{Yb}^{3+}-$ doped silicate glasses produced by low-dose carbon ion implantation. Jpn. J. Applied Phys., 51: 052601052604. DOI: 10.1143/JJAP.51.052601

Liu, C.X., T. Liu, X.H. Liu, W. Wei and B. Peng, 2011. Helium-implanted optical planar waveguides in $\mathrm{Nd}^{3+}$-doped phosphate glass. Chin. Phys. Lett., 28: 114205-114205. DOI: $10.1088 / 0256-$ 307X/28/11/114205

Liu, C.X., W.N. Li, W. Wei and B. Peng, 2012e. Optical planar waveguides in $\mathrm{Yb}^{3+}$-doped phosphate glasses produced by $\mathrm{He}^{+}$ion implantation. Chin. Phys. B, 21: 074211-074211. DOI: $10.1088 / 1674-$ $1056 / 21 / 7 / 074211$ 
Liu, K., E.Y.B. Puna, T.C. Sum, A.A. Bettiol and J.A.V. Kan et al., 2004. Erbium-doped waveguide amplifiers fabricated using focused proton beam writing. Applied Phys. Lett., 84: 684-686. DOI: 10.1063/1.1644922

Mairaj, A.K., A.M. Chard, D.P. Shepherd and D.W. Hewak, 2002. Laser performance and spectroscopic analysis of optically written channel waveguides in neodymium-doped gallium lanthanum sulphide glass. IEEE J. Sel. Top. Quantum Elect., 8: 13811388. DOI: 10.1109/JSTQE.2002.806672

Murphy, E.J., 1999. Integrated Optical Circuits and Components: Design and Applications. 1st Edn., CRC Press, New York, ISBN-10: 0824775775, pp: 468.

Najafi, S.I., T. Touam, R. Sara, M.P. Andrews and M.A. Fardad, 1998. Sol-Gel glass waveguide and grating on silicon. J. Lightwave Technol., 16: 1640-1646.

Nolte, S., M. Will, J. Burghoff and A. Tuennermann, 2003. Femtosecond waveguide writing: A new avenue to three-dimensional integrated optics. Applied Phys. A Mater. Sci. Process., 77: 109-111.

Okur, I. and P.D. Townsend, 1997. Waveguide formation by $\mathrm{He}^{+}$and $\mathrm{H}^{+}$ion implantation in filter glass containing nanoparticles. Nucl. Instrum. Methods Phys. Res. Sec. B: Beam Inter. Mater. Atoms, 124: 76-80. DOI: 10.1016/S0168-583X(97)00103-1

Psaila, N.D., R.R. Thomson, H.T. Bookey, A.K. Kar and N. Chiodo et al., 2007. Er:Yb-doped oxyfluoride silicate glass waveguide amplifier fabricated using femtosecond laser inscription. Applied Phys. Lett., 90: 131102-131104. DOI: 10.1063/1.2716866

Qiu, F. and T. Narusawa, 2010. Proton-implanted planar waveguide in gallium lanthanum sulphide glass. Jpn. J. Applied Phys., 49: 092503-092505. DOI: 10.1143/JJAP.49.092503

Ramaswamy, R.V. and R. Srivastava, 1988. Ionexchanged glass waveguides: A review. J. Lightw. Technol., 6: 984-1000. DOI: 10.1109/50.4090

Ramponi, R., R. Osellame and M. Marangoni, 2002. Two straightforward methods for the measurement of optical losses in planar waveguides. Rev. Sci. Instrum., 73: 1117-1121. DOI: 10.1063/1.1448143

Regener, R. and W. Sohler, 1985. Loss in low-finesse Ti: $\mathrm{LiNbO}_{3}$ optical waveguide resonators. Applied Phys. B, 36: 143-147. DOI: 10.1007/BF00691779

Righini, G.C., S. Pelli, M. Brenci, M. Ferrari and C. Duverger et al., 2001. Active optical waveguides based on Er- and Er/Yb-doped silicate glasses. J. Non-Cryst. Solids, 284: 223-229. DOI: 10.1016/S0022-3093(01)00406-9
Schineller, E.R., R.P. Flam and D.W. Wilmot, 1968. Optical waveguides formed by proton irradiation of fused silica. J. Opt. Soc. Am., 58: 1171-1173. DOI: 10.1364/JOSA.58.001171

Shepherd, D.P., D.J.B. Brinck, J. Wang, A.C. Tropper and D.C. Hanna et al., 1994. 1.9- $\mu$ m operation of a Tm:lead germanate glass waveguide laser. Opt. Lett., 19: 954-956. DOI: 10.1364/OL.19.000954

Sum, T.C., A.A. Bettiol, K. Liu, M.Q. Ren and E.Y.B. Pun et al., 2005. Proton beam writing of erbiumdoped waveguide amplifiers. Nucl. Instru. Methods Phys. Res. Sec. B: Beam Inter. Mater. Atoms, 231: 394-399. DOI: 10.1016/j.nimb.2005.01.089

Svecova, B., J. Spirkova, S. Janakova and M. Mika, 2008. Ion-exchanged optical waveguides fabricated in novel $\mathrm{Er}^{3+}$ and $\mathrm{Er}^{3+} / \mathrm{Yb}^{3+}$-doped silicate glasses: Relations between glass composition, basicity and waveguide properties. Mater. Sci. Eng.: B, 149: 177180. DOI: 10.1016/j.mseb.2007.11.019

Tan, Y., F. Chen, D. Jaque, W.L. Gao and H.J. Zhang et al., 2009. Ion-implanted optical-stripe waveguides in neodymium-doped calcium barium niobate crystals. Opt. Lett., 34: 1438-1440. DOI: 10.1364/OL.34.001438

Tan, Y., F. Chen, L.L. Hu, P.F. Xing and Y.X. Chen et al., 2007. Ridge optical waveguide in an $\mathrm{Er}^{3+} / \mathrm{Yb}^{3+}$ codoped phosphate glass produced by $\mathrm{He}^{+}$ion implantation combined with $\mathrm{Ar}^{+}$ion beam etching. J. Phys. D: Applied Phys., 40: 6545-6548. DOI: 10.1088/0022-3727/40/21/013

Townsend, P. and J. Olivares, 1997. Laser processing of insulator surfaces. Applied Surf. Sci., 109-110: 275282. DOI: 10.1016/S0169-4332(96)00621-6

Townsend, P.D., P.J. Chandler and L. Zhang, 1994. Optical Effects of Ion Implantation. 1st Edn., Cambridge University Press, Cambridge, ISBN-10: 0521394309, pp: 296.

Valle, G.D., R. Osellame, N. Chiodo, S. Taccheo and G. Cerullo et al., 2005. C-band waveguide amplifier produced by femtosecond laser writing. Opt. Exp., 13: 5976-5982. DOI: 10.1364/OPEX.13.005976

Veasey, D.L., D.S. Funk, N.A. Stanford and J.S. Hayden, 1999. Arrays of distributed-Bragg-reflector waveguide lasers at $1536 \mathrm{~nm}$ in $\mathrm{Yb} / \mathrm{Er}$ codoped phosphate glass. Applied Phys. Lett., 74: 789-791. DOI: $10.1063 / 1.123368$

Veasey, D.L., D.S. Funk, P.M. Peters, N.A. Sanford and G.E. Obarski et al., 2000. Yb/Er-codoped and Ybdoped waveguide lasers in phosphate glass. J. NonCryst. Solids, 263-264: 369-381. DOI: 10.1016/S0022-3093(99)00677-8 
Vezenov, D.V., B.T. Mayers, R.S. Conroy, G.M. Whitesides and P.T. Snee et al., 2005. A lowthreshold, high-efficiency microfluidic waveguide laser. J. Am. Chem. Soc., 127: 8952-8953. DOI: 10.1021/ja0517421

Wang, L., F. Chen, X.L. Wang, K.M. Wang and Y. Jiao et al., 2007. Low-loss planar and stripe waveguides in $\mathrm{Nd}^{3+}$-doped silicate glass produced by oxygen-ion implantation. J. Applied Phys., 101: 053112-053115. DOI: $10.1063 / 1.2710293$

Wang, X.L., C.X. Liu, J.H. Zhao and H.J. Zhang Liu, 2009. Property studies of optical waveguide formed by IIkeV He-ion implanted into a Nd:CNGG crystal. J. Korean Phys. Soc., 55: 2638-2641. DOI: 10.3938/jkps.55.2638

Wang, X.L., K.M. Wang, G. Fu, S.L. Li and D.Y. Shen et al., 2004. Low propagation loss of the waveguides in fused quartz by oxygen ion implantation: Errata. Opt. Express, 12: 5285-5286. DOI: 10.1364/OPEX.12.005285
White, J.M. and P.F. Heidrich, 1976. Optical waveguide refractive index profiles determined from measurement of mode indices: A simple analysis. Applied Opt., 15: 151-155. DOI: 10.1364/AO.15.000151

Zhang, S.M., K.M. Wang, X.Z. Liu, Z.F. Bi and X.H. Liu, 2010. Planar and ridge waveguides formed in $\mathrm{LiNbO}_{3}$ by proton exchange combined with oxygen ion implantation. Opt. Exp., 18: 15609-15617. DOI: 10.1364/OE.18.015609

Zhao, R.T., L.F. Shen and H. Lin, 2011. Gain evaluation of compact designing on $\mathrm{Er}^{3+} / \mathrm{Yb}^{3+}$ codoped germanate glass channel waveguide. Optik-Int. J. Light Elect. Opt., 122: 2237-2241. DOI: 10.1016/j.ijleo.2011.02.017

Zhou, Z., A. Lin, H. Guo, X. Liu and C. Hou et al., 2010. $\mathrm{Tb}^{3+} / \mathrm{Yb}^{3+}$ heavily-doped tellurite glasses with efficient green light emission. J. Non-Cryst. Solids, 356: 2896-2899.

10.1016/j.jnoncrysol.2010.09.010 